\title{
Retraction Note to: METADEPCAS: Introducing Semantic RFID Data Management
}

Ismael Abad, Carlos Cerrada, and José Antonio Cerrada

\author{
Retraction Note to: \\ Chapter "METADEPCAS: Introducing Semantic RFID \\ Data Management" in: G. Urzaiz et al. (Eds.): Ubiquitous \\ Computing and Ambient Intelligence, LNCS 8276, \\ https://doi.org/10.1007/978-3-319-03176-7_26
}

\begin{abstract}
"The authors have retracted this chapter [1] because it shows significant overlap with a previous publication by De Virgilio et al [2]. All authors agree to this retraction.

1. Abad, I., Cerrada, C., Cerrada, J.A.: METADEPCAS: Introducing Semantic RFID Data Management. In: Urzaiz, G., Ochoa, S.F., Bravo, J., Chen, L.L., Oliveira, J. (eds.) UCAmI 2013. LNCS, vol. 8276, pp. 199-206, Springer International Publishing Switzerland (2013). https://doi.org/10.1007/978-3-319-03176-7_26

2. De Virgilio, R., Di Sciascio, E., Ruta, M., Scioscia, F., Torlone, R.: Semantic-Based RFID Data Management. In: Ranasinghe, D.C., Sheng, Q.Z., Zeadally, S. (eds) Unique Radio Innovation for the 21st Century, pp. 111-141, Springer-Verlag Berlin Heidelberg (2010). https://doi.org/10.1007/978-3-642-03462-6_6”"
\end{abstract}

\footnotetext{
The retracted version of this chapter can be found at https://doi.org/10.1007/978-3-319-03176-7_26 